\title{
Important steps in a Correlative Light Electron Microscopy Experiment
}

\author{
Paul Verkade
}

Wolfson Bioimaging Facility, Schools of Biochemistry and Physiology \& Pharmacology, Medical Sciences Building, University Walk, University of Bristol, Bristol, UK E-mail: p.verkade@bristol.ac.uk

Correlative Light Electron Microscopy combines the strengths of light and electron microscopy in one experiment and the sum of such an experiment should provide more data / insight than each technique alone $(1+1=3)$. There are many ways to perform a CLEM experiment and a variety of microscopy modalities can be combined. The choice of these instruments and the experimental approach should primarily depend on the scientific question to be answered.

Any CLEM experiment can usually be divided in 3 parts; probes, processing, and analysis. I will discuss 3 processing techniques based on light microscopy in conjunction with Transmission Electron Microscopy, each with its specific application and its advantages and challenges.

1. The first is based on the use of coverslips with an embossed finder pattern. Importantly, it allows for live cell imaging and captures an event of interest using chemical fixation (Figure 1) [1]. This is possibly one of the technically easiest CLEM techniques but retracing the same object in the light and electron microscope remains a challenge. Hence we have focussed on developing algorithms that allow confident retracing and identification of the same structure observed in the light microscope to be found in the electron microscope.

2. A second uses the Tokuyasu cryo immuno labelling technique to trace back objects of interest (Figure 2) [2]. This allows for relatively high immuno labelling efficiencies but is almost impossible in combination with live cell imaging.

3. The third is based on cryo-fixation to obtain best possible preservation of ultrastructure $[3,4]$. This allows us to capture events that would be lost because of chemical fixation, e.g. membrane tubules. It allows for live cell imaging but immuno labelling options are limited.

\section{REFERENCES.}

[1] Hodgson L, D. Nam, J. Mantell, A. Achim, and P. Verkade, Meth. Cell Biol. 124 (2014), p. 1.

[2] Hodgson L, J. Tavaré, and P. Verkade, Protoplasma 251 (2014) p. 403.

[3] Brown, E., J., Van Weering, T. Sharp, J. Mantell, and P. Verkade, Meth. Cell Biol. 111 (2012), p. 175.

[4] P. Verkade, J. Microsc. (Oxford) 230, p. 317. 

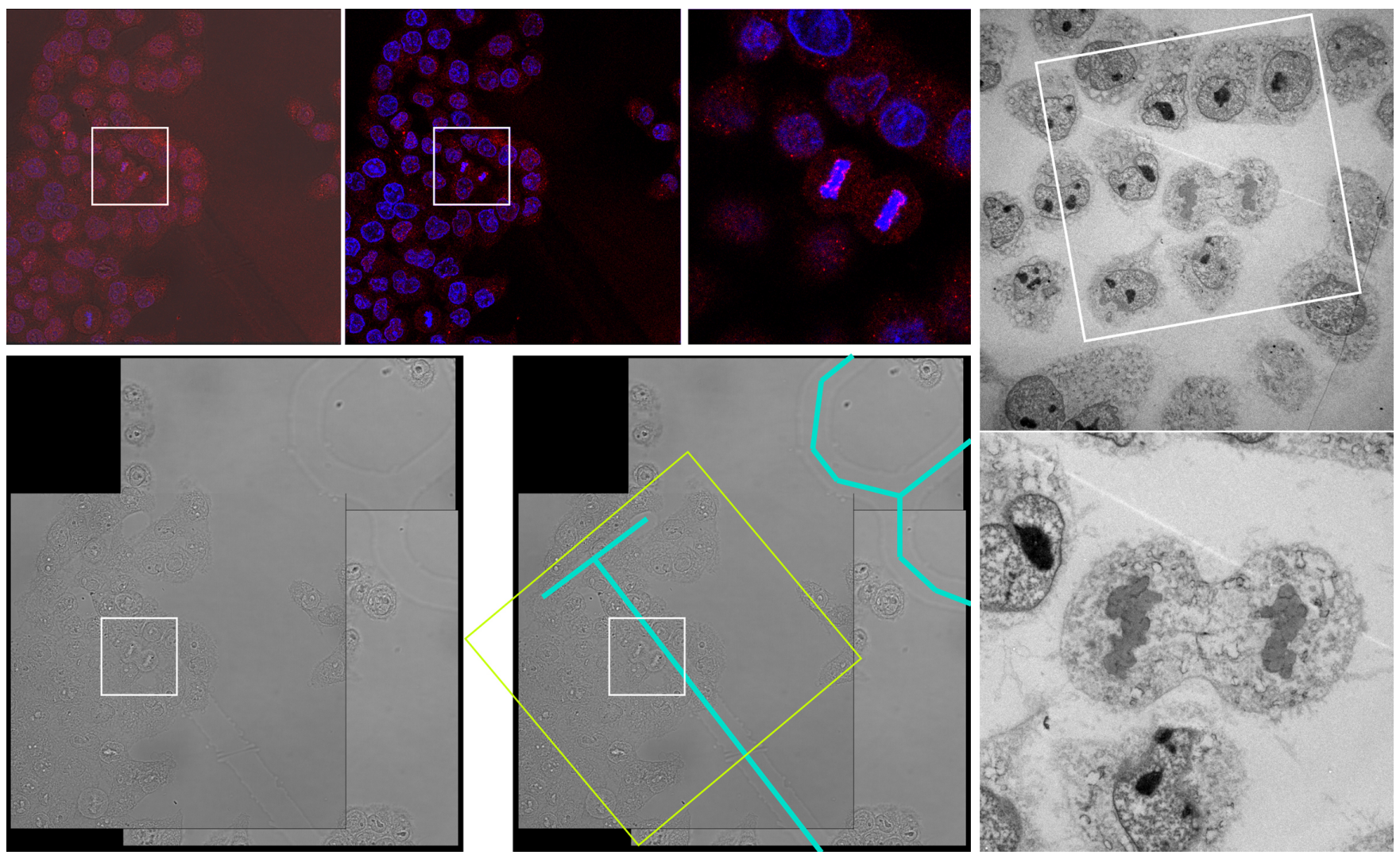

Figure 1. CLEM allows the identification and subsequent high-resolution analysis of 1 special cell amongst 100s. In the top left row, a dividing cell $(1$ in $>100)$ is identified based on its DNA staining (blue). With the aid of embossed coverslips (for full details see [1]) the dividing cell can be traced back in the EM and studied at higher resolution.
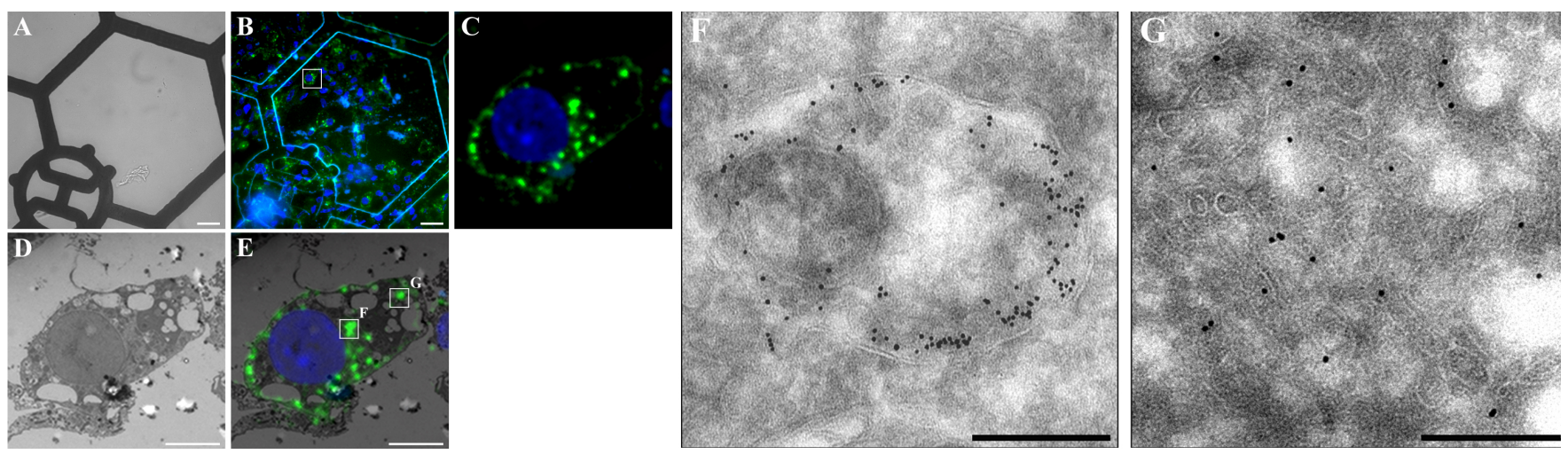

Figure 2. CLEM using Tokuyasu cryo immuno gold labelling, an excellent way to zoom into specific structures with high labelling efficiency. Modified from [2]. 